\title{
PROBLEMS WITH MAXIMUM ENTROPY
}

\author{
J.E.B. Ponsonby \\ University of Manchester \\ Jodre11 Bank, United Kingdom.
}

ABSTRACT

Two rival expressions for entropy have been used in maximum entropy image formation. Attention is drawn to the fact that both have been shown to be limiting cases of a general expression for the entropy of the photon distribution swept up during an observation. All measures of entropy are based on some physical model of the radiation mechanism. It is argued that knowledge of its character should be fully included in the image formation process. 